\title{
Gender Effects on Acute Heart Failure
}

\author{
Arnon Blum ${ }^{1 *}$, Rizak Sirchan ${ }^{1}$, Lital Keinan-Boker ${ }^{2,3}$ \\ ${ }^{1}$ Department of Medicine, Baruch-Padeh Poria Medical Center, Tiberias, Israel; ${ }^{2}$ Israel Center for Disease Control, Ministry of \\ Health, Jerusalem, Israel; ${ }^{3}$ School of Public Health, University of Haifa, Haifa, Israel. \\ Email: *ablum@poria.health.gov.il
}

Received March 10 ${ }^{\text {th }}, 2011$; revised April 19 ${ }^{\text {th }}, 2011$; accepted May $3^{\text {rd }}, 2011$.

\begin{abstract}
Background: congestive heart failure is the leading cause of hospitalization in the elderly. Little is known about gender effect on baseline characteristics and in-hospital outcome in patients admitted with acute heart failure. Our purpose was to study the gender effect on in-hospital mortality in acute heart failure patients. Methods and Results: a prospective study [143 patients, 67 men (73.9 \pm 13.8 years old $)$ and 76 women $(77.8 \pm 10.1$ years old $)(p=0.059)]$ followed in-hospital outcome of patients with acute heart failure admitted to the hospital. Clinical parameters included body mass index $(B M I)$, ankle brachial index $(A B I)$, left ventricular ejection fraction $(L V E F)$, re-admissions within 1 year, and in-hospital mortality. The gender effects that were studied included height, BMI, smoking, coronary artery disease, LVEF and mortality: in total, 9 (6.3\%) patients died, of them 8 (10.5\%) women and 1 (1.5\%) man. Women were shorter $(p<0.001)$, had a higher BMI $(p=0.053)$, reported less frequently on current smoking $(p<0.001)$, had lower prevalence of coronary artery disease $(p=0.016)$, had a better LVEF $(p=0.02)$, but still, had a higher mortality rate $(p=$ 0.026). The only variables independently affecting in-hospital mortality in women were height and recurrent admissions. When we tested for the effect of height and recurrent admissions on mortality only among females by a multivariate analysis height inversely and independently affected in-hospital mortality $(p=0.024)$, as well as recurrent admissions ( $p=0.031)$. Conclusions: in-hospital mortality was significantly higher in women compared with men admitted with acute heart failure. Among females, the only independent variables that affected mortality were low stature and recurrent admissions.
\end{abstract}

Keywords: Gender, Heart Failure, in Hospital Death

\section{Introduction}

Congestive heart failure (CHF) is the leading cause of hospitalizations in the elderly. Little is known about gender differences in baseline characteristics and outcomes in patients with acute congestive heart failure. The epidemic of cardiovascular disease in women has attained increasing recognition.

The Framingham heart study reported an estimated prevalence of heart failure of $0.8 \%$ in both genders within the age group of 50 - 59 years. The prevalence increases notably with advancing age, rising to $6.6 \%$ and $7.9 \%$ in men and women, respectively, aged $80-89$ years [1]. A study from Olmsted County, Minnesota, has evaluated the prevalence of "diastolic" heart failure. In this survey of the population aged over 45 years, the overall prevalence of heart failure was $2.2 \%, 44 \%$ of heart failure cases had preserved systolic function (LVEF $>50 \%$ ). The prevalence of diastolic dysfunction was higher in the older population and in those with a history of hypertension, diabetes, coronary disease and previous myocardial infarction. The prevalence of diastolic dysfunction was similar in both genders [2].

According to the Framingham study, the incidence of heart failure was significantly higher in men compared to women at all ages, with an age-standardised incidence ratio of 1.67 [1]. Coronary artery disease was reported as the etiological factor in $59 \%$ of men and $47 \%$ of women for the period 1948-1988. Valvular disease, hypertension, and diabetes were more common in women than in men [1].

The incidence and prevalence of heart failure is lower in women than men at all ages. However, due to the steep increase in incidence with age, and the larger population of elderly women in the developed world, the total number of men and women living with heart failure is similar. Heart failure with preserved systolic function ("diastolic" dysfunction) is more common in women, perhaps related to gender differences in the myocardial response to injury, and the lower prevalence of coronary 
artery disease in women at all ages compared with men [3].

In this prospective study we addressed some of the questions that were raised about gender and acute heart failure during in-hospital stay-clinical characteristics and in hospital outcome. Our purpose was to study the in hospital outcome of patients that were admitted with acute heart failure.

\section{Methods}

This was a prospective, hospital-based study. The study population included 143 consecutive patients that were admitted with acute heart failure to the Medicine Ward in the Baruch-Padeh Poria Medical Center during the years 2008-2010.

The inclusion criteria included females and male patients older than 18 years old with no known chronic or acute infectious, inflammatory or autoimmune disease. The exclusion criteria included patients younger than 18 years old, patients who also had an active cancer or an autoimmune, inflammatory or an infectious disease.

All had an exacerbation of a known heart failure and were treated accordingly by beta blockers, angiotensin converting enzyme inhibitors, and diuretics as needed. On admission, all participants had their weight and height measured and their body mass index (BMI) was calculated. Their peripheral artery disease was evaluated by measuring the ankle-brachial index (ABI); the abdominal circumference served as a proxy for central obesity, and the cardiac function was evaluated by measuring the ejection fraction (echocardiography).

In addition, information on clinical parameters like current smoking (self report), alcohol use, prevalence of Atrial Fibrillation or other arrhythmias, Diabetes Mellitus type II, renal function, Hypertension, documented Coronary Artery Disease (CAD), and Rheumatic Valvular Heart Disease (documented by echocardiography) was collected.

Follow up continued during hospitalization.

The primary end point was to study the gender effect on in-hospital mortality.

\section{Statistical Analysis}

Gender differences in categorical variables were assessed by using the Chi-square test, and in continuous variables, by using the independent sample $T$-test. Univariate analyses, where in-hospital mortality served as the dependent variable, were used to identify significant correlates. Multivariate analyses, using logistic regression models, were stratified by gender and included variables that significantly affected in-hospital mortality in univariate analyses. $P$-value was set at 0.05 , and all analyses were two-tailed. The analyses were carried out using the Statistical Package for Social Sciences (SPSS), version 17.

\section{Results}

One hundred and forty three acute heart failure patients were admitted to our Medicine Department. There were 67 men (mean age: $73.9 \pm 13.8$ years old; range: 23 - 99) and 76 women (mean age: $77.8 \pm 10.1$ years old; range: 55 - 96) ( $p=0.059)$. Men tended to report on current smoking more frequently than women (31 men vs. 6 women; $p<0.001$ ), were taller (mean height: $1.69 \pm 0.1$ meter vs. $1.58 \pm 0.1 ; p<0.001$ ), and had a lower BMI (mean BMI: $28.4 \pm 5.9$ vs. $30.8 \pm 8.7 ; p=0.053$ ) (Table 1). Coronary artery disease was more prevalent among men (42 [68\%] vs. 36 [47\%] in women; $p=0.016)$, and their ejection fraction was lower (48.2 \pm 15.7 vs. $55.1 \pm$ 13.9 in women; $p=0.020$ ) (Table 1).

No difference was observed between genders in relation to prevalence of diabetes mellitus, hypertension, rheumatic valvular heart disease, abdominal circumference and ankle brachial index (Table 1), but women had a trend towards a higher prevalence of recurrent hospitalizations (8[11\%] vs. 2[3\%], $p=0.078$ ), and a lower prevalence of peripheral artery disease (ABI less than $0.9,22[33 \%]$ among men, 18[24\%] among women; $p=$ 0.224) (Table 1).

There were 8 in-hospital deaths among women and only 1 in-hospital death among men $(p=0.026)$ (Table 1). Variables that were significantly associated with in-hospital mortality included gender (female), recurrent hospitalizations, height (lower) and weight (lower) (Table 2). Age, current smoking, BMI, LVEF, and prevalence of comorbidites (atrial fibrillation, diabetes mellitus, hypertension, coronary artery disease and valvular rheumatic heart disease) did not seem to significantly

Table 1. Clinical characteristics of the study population.

\begin{tabular}{lllll}
\hline & Total & Males & Females & p-value \\
\hline Gender & $143(100 \%)$ & $67(47 \%)$ & $76(53 \%)$ & \\
Age & $76.1 \pm 12.0$ & $73.9 \pm 13.8$ & $77.8 \pm 10.1$ & 0.059 \\
Weight (Kg) & $78.6 \pm 20.1$ & $80.9 \pm 17.7$ & $76.5 \pm 21.8$ & 0.187 \\
Height (M) & $1.63 \pm 0.1$ & $1.69 \pm 0.1$ & $1.58 \pm 0.1$ & $<0.001$ \\
BMI & $29.6 \pm 7.6$ & $28.4 \pm 5.9$ & $30.8 \pm 8.7$ & 0.053 \\
Abd Circ (cm) & $108.4 \pm 20.9$ & $108.3 \pm 19.7$ & $108.5 \pm 22.0$ & 0.951 \\
ABI & $0.97 \pm 0.29$ & $0.98 \pm 0.33$ & $0.97 \pm 0.24$ & 0.764 \\
Smokers & $37(27 \%)$ & $31(50 \%)$ & $6(8 \%)$ & $<0.001$ \\
DM type II & $77(56 \%)$ & $35(56 \%)$ & $42(55 \%)$ & 0.889 \\
HTN & $119(86 \%)$ & $51(82 \%)$ & $68(89 \%)$ & 0.221 \\
CAD & $78(56 \%)$ & $42(68 \%)$ & $36(47 \%)$ & 0.016 \\
RHD & $54(39 \%)$ & $21(34 \%)$ & $33(43 \%)$ & 0.253 \\
A. Fibrillation & $57(42 \%)$ & $24(39 \%)$ & $33(43 \%)$ & 0.630 \\
LVEF (\%) & $51.9 \pm 15.1$ & $48.2 \pm 15.7$ & $55.1 \pm 13.9$ & 0.020 \\
Recurrent & & & & \\
Admissions & $10(7 \%)$ & $2(3 \%)$ & $8(11 \%)$ & 0.078 \\
In-Hospital Death & $9(6 \%)$ & $1(2 \%)$ & $8(11 \%)$ & 0.026 \\
\hline
\end{tabular}


Table 2. Correlates of in-hospital mortality-univariate analyses.

\begin{tabular}{lcll}
\hline Variable & Mortality+ & Mortality- & $p$-value \\
\hline $\begin{array}{l}\text { Gender }(n=143) \\
\text { Males }\end{array}$ & $1(1.5 \%)$ & $66(98.5 \%)$ & 0.026 \\
Females & $8(10.5 \%)$ & $68(89.5 \%)$ & \\
Recurrent Hospitalizations $(n=143)$ & & \\
Yes & $4(40 \%)$ & $6(60 \%)$ & $<0.001$ \\
No & $5(3.8 \%)$ & $128(96.2 \%)$ & \\
Continuous Variables & & & \\
Height $(\mathrm{M})(n=141)$ & $1.50 \pm 0.07$ & $1.63 \pm 0.08$ & $<0.001$ \\
Weight $(\mathrm{Kg})(n=140)$ & $63.00 \pm 24.55$ & $79.41 \pm 19.61$ & 0.035 \\
\hline
\end{tabular}

affect the outcome.

When stratified by gender, the only significant factor in men was recurrent hospitalizations $(p<0.001)$. In women, significant correlates affecting in-hospital mortality included recurrent hospitalizations $(p=0.009)$, low ABI $<0.9(p=0.006)$, and being shorter $(p=$ 0.006 ).

We used a logistic regression model to assess the independent effect of each of these variables on in-hospital mortality in women. Only height $(\mathrm{OR}=0.000,95 \% \mathrm{CI}$ $0.000-0.116, p=0.024$ ) and recurrent admissions (OR $=9.290,95 \%$ CI $1.229-70.220, p=0.031$ ) remained statistically significant (Table 3). The model was not applied for men since only one man died.

\section{Discussion}

Of all the cardiovascular diseases, the prevalence of heart failure is the only one that continues to increase in the United States [4]. Heart failure affects mainly the elderly, the prevalence and incidence of heart failure are expected to increase over the next several decades. Gender differences in the syndrome of heart failure occur in etiology and pathophysiology and lead to differences in the clinical presentation and course of the syndrome. Hypertension and diabetes play a major role as causes of heart failure in women [5]. Major risk factors for heart failure have been evaluated in the past 50 years in the Framingham heart study program. Hypertension carried the largest population-attributable risk factor for heart failure, underlying $59 \%$ of heart failure cases in women and $39 \%$ in men with a hazard ratio of 3.35 in women and 2.07 in men [6]. A particularly steep increase in the prevalence of hypertension with increasing

Table 3. Logistic regression model to assess the independent effect of each of these variables on in-hospital mortality in women.

\begin{tabular}{llll}
\hline Variable & OR & 95\% CI & P-value \\
\hline Recurrent patient (ref: no) & 9.290 & $1.229-70.220$ & 0.031 \\
Height in cm (continuous) & 0.000 & $0.000-0.116$ & 0.024 \\
\hline
\end{tabular}

age in women compared with men has also been observed in a European WHO-MONICA cohort [7]. Myocardial cell death, apoptosis, and cellular hypertrophy of the remaining cells are more pronounced in the male than in the female myocardium [8,9]. Female patients with aortic stenosis exhibit relatively less ventricular dilatation and less myocardial hypertrophy and have better preserved left ventricular function at a comparable degree of stenosis than male patients [9].

Adipose tissue produces inflammatory mediators that increase the risk for cardiovascular disease and diabetes [10]. Adipocytokines (interleukin-1 $\beta$, interleukin 6, leptin, tumor necrosis factor $\alpha$ ) and adiponectin levels exhibit large sexual dimorphisms. Fat tissue expresses aromatase, and conversion of testosterone to estrogen by aromatase is one of the main sources for estrogen in postmenopausal women [5].

The primary end point of our study was the gender effect on in-hospital mortality, and the secondary end points were to study the gender effect on recurrent hospitalizations and peripheral artery disease (measured by ABI of less than 0.9).

In our study there were much more in-hospital deaths among women. Variables that were significantly associated with in-hospital mortality included gender (female patients), recurrent hospitalizations, height (lower height -more deaths), and weight (lower weights-more deaths). The only significant factor in men was recurrent hospitalizations, while in women increased death rates were affected by recurrent hospitalizations, peripheral artery disease (ABI lower than 0.9) and a low stature. After using a logistic regression analysis only height remained statistically significant.

A prospective study evaluated gender differences among 217 patients presenting with acute heart failure to the emergency department in Switzerland. The primary end point was all-cause mortality. Women were older and had less pulmonary co-morbidity, more jugular venous pressure and higher diastolic blood pressure and troponin level at presentation; however, the trend toward lower survival in women seemed primarily related to higher age rather than gender itself. Female sex was not found as an independent predictor of long-term mortality in acute heart failure [11]. Another study (a retrospective one) did not find any significant gender differences in the initial presentation, disposition, and diagnostic testing of patients with acute heart failure admitted through the emergency department [12].

The impact of gender was assessed in the beta blocker evaluation of survival trial (BEST) which randomized 2 708 patients with NYHA class III/IV and LVEF less than $35 \%$ to bucindolol versus placebo. Significant differences in baseline clinical and laboratory characteris- 
tics were found. Compared to men, women were younger, more likely to be black, had a higher prevalence of non-ischemic etiology, had higher right and left ventricular ejection fraction, higher heart rate, greater cardiothoracic ratio, higher prevalence of left bundle branch block, lower prevalence of atrial fibrillation, and lower plasma nor-epinephrine level. Coronary artery disease and LVEF appeared to be stronger predictors of prognosis in women; however, in the non-ischemic patients, women had a significantly better survival rate compared with men [13].

Obesity could be a factor in prognosis in heart failure patients; however, studies have shown a better survival in heart failure patients with decreased LVEF and a higher BMI compared to those with a lower BMI [14-19]. Another study has demonstrated an increased mortality in patients with heart failure and preserved systolic function and low BMI; however, with a BMI > 45 mortality increased, raising the possibility of a $U$ shaped relationship between BMI and survival [20]. A meta-analysis of 9 retrospective studies (mean follow-up of 2.7 years) found that compared to individuals without elevated BMI, both overweight and obese patients had a lower all-cause mortality and also lower cardiovascular mortality. In a risk-adjusted sensitivity analysis, both obesity and overweight remained protective against mortality [21].

In our study a gender effect was observed on BMI; women had a higher BMI compared with men (30.0 \pm 8.7 vs. $28.4 \pm 5.9, p=0.053$ ) —but such an effect should have been a protective factor according to recent studies on BMI and weight in patients with heart failure [20, 21], but in our study obesity and overweight did not serve as a protective factor against in-hospital mortality and had no advantage for women.

Another interesting finding was the gender effect on height; women were significantly shorter than men with acute heart failure $(1.58 \pm 0.1 \mathrm{~m}$ vs. $1.69 \pm 0.1 \mathrm{~m}, p<$ 0.001).

An autopsy Finish study evaluated coronary atherosclerosis among 599 females that died a sudden death and found that $50 \%$ of women over 41 years old had coronary lesions, $32 \%$ in women from 31 to 40 years, $17 \%$ in women from 21 to 30, and 6\% in women under 20 years. Coronary atherosclerosis was correlated with short stature as well as with BMI and abdominal fat [22]. A retrospective meta-analysis assessed the relationship between short stature and coronary heart disease from 52 studies (3,012,747 individuals). The short ones were below $160.5 \mathrm{~cm}$ and the tall ones over $173.9 \mathrm{~cm}$ on average. Among the shortest height category, the relative risks were 1.35 for all-cause mortality, 1.55 for all cardiovascular disease mortality, 1.49 for coronary heart disease, and 1.52 for myocardial infarction when compared with those within the highest height category. Short stature was associated with increased cardiovascular morbidity and mortality in both genders. No gender effect was observed in this study. Only height was the independent parameter that affected death [23]. On the other hand, a prospective study of women hospitalized with acute coronary event (292 Swedish women, aged 65 years or younger, follow-up 4.8 years) found that independent of the confounding effects of other risk factors of clinical importance for CHD the shortest 25\% of women $(<160 \mathrm{~cm})$ had a 2.1-fold increased rate of developing adverse cardiac events compared with the tallest 25\% (>165 cm) [24].

To examine the influence of race and gender on hospital outcome for patients with congestive heart failure a retrospective study examined records on all $1995 \mathrm{New}$ York State hospital discharges assigned ICD-9-CM codes indicative of this diagnosis. It was found that 45,894 patients (black women, 4750; black men, 3370; white women, 21,165; white men, 16,609) had were admitted with congestive heart failure. Blacks underwent noninvasive cardiac procedures more often than whites; procedure and specialty use rates were lower among women than among men. After adjusting for other patient characteristics and hospital type and location, we found race to be an important determinant of length of stay in the hospital (black, 10.4 days; white, 9.3 days; $p=0.0001$ ), hospital charges (black, $\$ 13,711$; white, $\$ 11,074 ; p=0.0001$ ), mortality (black-to-white odds ratio $=0.832 ; p=0.003)$, and readmission (black-to-white odds ratio $=1.301 ; p=0.0001)$. Gender was an important determinant of length of stay (women, 9.8 days; men, 9.2 days; $p=0.0001$ ), hospital charges (women, \$11,690; men, \$11,348; $p=0.02$ ), and mortality (women-to-men odds ratio $=0.878 ; p=0.0008$ ). The conclusion of that study was that race and gender affect care process and hospital-based case outcomes for patients with CHF [25].

\section{Conclusions}

Our study has demonstrated a gender effect on in-hospital mortality among acute heart failure patients. The only epidemiological data that could help explain our results are studies that have demonstrated a link between short stature and cardiovascular morbidity and mortality. Our female population was about $10 \mathrm{~cm}$ shorter compared with men, and this significant difference may explain our results.

The novelty of our study was that it was a prospective study that evaluated gender effect on in-hospital mortality in patients admitted with acute heart failure-unlike any other study that examined these specific parameters 
in patients admitted with acute heart failure.

\section{Study Limitation}

The small sample size is limiting our ability to have definite conclusions. We believe that by enlarging the population of heart failure patients we will be able to have more solid conclusions. Our plan is to continue with this study and to recruit many more patients with acute heart failure.

\section{Conflict of Interest Disclosures}

NONE for all authors.

\section{REFERENCES}

[1] K. K. Ho, J. L. Pinsky, W. B. Kannel and D. Levy, "The Epidemiology of Heart Failure: The Framingham Study," Journal of the American College of Cardiology, Vol. 22, No. 4, 1993, pp. 6A-13A. doi:10.1016/0735-1097(93)90455-A

[2] M. M. Redfield, S. J. Jacobsen, J. C. Burnett Jr., D. W. Mahoney, K. R. Bailey and R. J. Rodeheffer, "Burden of Systolic and Diastolic Ventricular Dysfunction in the Community: Appreciating the Scope of the Heart Failure Epidemic," The Journal of the American Medical Association, Vol. 289, No. 2, 2003, pp. 194-202. doi:10.1001/jama.289.2.194

[3] P. A. Mehta and M. R. Cowie, "Gender and Heart Failure: A Population Perspective,” Heart, Vol. 92, No. 3, 2006, pp. 14-18. doi:10.1136/hrt.2005.070342

[4] K. F. Adams Jr., "New Epidemiologic Perspectives Concerning Mild-to-Moderate Heart Failure,” The American Journal of Medicine, Vol. 110, No. 7, 2001, pp. 6S-13S. doi:10.1016/S0002-9343(98)00383-0

[5] V. Regitz-Zagrosek, E. Lehmkuhl, H. B. Lehmkuhl and R. Hetzer, "Gender Aspects in Heart Failure," Archives Des Maladies du Coeur Et Des Vaisseaux, Vol. 97, No. 9, 2004, pp. 1-10.

[6] D. Levy, M. G. Larson, R. S. Vason, W. B. Kannel and K. K. Ho, "The Progression from Hypertension to Congestive Heart Failure,” The Journal of the American Medical Association, Vol. 275, No. 20, 1996, pp. 1557-1562. doi:10.1001/jama.275.20.1557

[7] C. Gasse, H. W. Hense, J. Stieber, A. Doring, A. D. Liese and U. Keil, "Assessing Hypertension Management in the Community: Trends of Prevalence, Detection, Treatment, and Control of Hypertension in the MONICA Project, Augsburg 1984-1995," Journal of Human Hypertension, Vol. 15, No. 1, 2001, pp. 27-36. doi:10.1038/sj.jhh.1001120

[8] S. Guerra, A. Leri, X. Wang, N. Finato, C. Di Loreto, C. A. Beltrami, J. Kajstura and P. Anversa, "Myocyte Death in the Failing Human Heart Is Gender Dependent," Circulation Research, Vol. 85, No. 9, 1999, pp. 856-866.

[9] J. D. Carroll, E. P. Carroll, T. Feldman, D. M. Ward, R. M. Lang, D. McGaughey and R. B. Karp, "Sex Associated Differences in Left Ventricular Function in Aortic
Stenosis of the Elderly," Circulation, Vol. 86, No. 4, 1992, pp. 1099-1107.

[10] K. E. Wellen and G. S. Hotamisligil, "Obesity Induced Inflammatory Changes in Adipose Tissue,” The Journal of Clinical Investigation, Vol. 112, No. 12, 2003, pp. 1785-1788.

[11] M. Ritter, K. Laule-Kilian, T. Klima, A. Christ, M. Christ, A. Perruchoud and C. Mueller, "Gender Differences in Acute Congestive Heart Failure," Swiss Medical Weekly, Vol. 136, No. 19-20, 2006, pp. 311-317.

[12] H. M. Prendergast, K. Reddy, M. B. Latayan, E. B. Bunney and A. Schlichting, "Gender Differences in Presentation, Management and Disposition of Heart Failure Patients in the Emergency Setting," The Internet Journal of emergency Medicine, Vol. 2, No. 1, 2004, pp. 1-8.

[13] J. K. Ghali, H. J. Krause-Steinrauf, K. F. Adams, S. S. Khan, Y. D. Rosenberg, C. W. Yancy, J. B. Young, S. Goldman, M. A. Peberdry and J. Lindenfeld, “Gender Differences in Advanced Heart Failure: Insights from the BEST Study," Journal of the American College of Cardiology, Vol. 42, 2003, pp. 2128-2134. doi:10.1016/j.jacc.2003.05.012

[14] T. B. Horwich, G. C. Fonarow, M. A. Hamilton, W. R. MacLellan, M. A. Woo and J. H. Tillisch, "The RelationShip between Obesity and Mortality in Patients with Heart Failure,” Journal of American College of Cardiology, Vol. 38, No. 3, 2001, pp. 789-795.

[15] A. F. Osman, M. R. Mehra, C. J. Lavie, E. Nunez and R. V. Milani, "The Incremental Prognostic Importance of Body Fat Adjusted Peak Oxygen Consumption in Chronic Heart Failure,” Journal of American College of Cardiology, Vol. 36, No. 7, 2000, pp. 2126-2131.

[16] L. W. Lissin, A. J. Gauri, V. F. Froelicher, A. Ghayoumi, J. Myers and J. Giacommini, "The Prognostic Value of Body Mass Index and Standard Exercise Testing in Male Veterans with Congestive Heart Failure,” Journal of Cardiac Failure, Vol. 8, No. 4, 2002, pp. 206-215. doi:10.1054/jcaf.2002.126812

[17] C. H. Davos, W. Doehner, M. Rauchhaus, M. Cicoira, D. P. Francis, A. J. Coats, A. L. Clark and S. D. Anker, "Body Mass and Survival in Patients with Chronic Heart Failure without Cachexia: The Importance of Obesity," Journal of Cardiac Failure, Vol. 9, No. 1, 2003, pp. 29-35. doi:10.1054/jcaf.2003.4

[18] A. Mosterd, B. Cost, A. W. Hoes, M. C. De Bruijne, J. W. Deckers, A. Hofman and D. E. Grobbee, "The Prognosis of Heart Failure in the General Population," European Heart Journal, Vol. 22, No. 15, 2001, pp. 1318-1327. doi:10.1053/euhj.2000.2533

[19] C. J. Lavie, A. F. Osman, R. V. Milani and M. R. Mehra, "Body Composition and Prognosis in Chroniv Systolic Heart Failure: The Obesity Paradox,” American Journal of Cardiology, Vol. 91, No. 7, 2003, pp. 891-894. doi:10.1016/S0002-9149(03)00031-6

[20] J. R. Kapoor and P. A. Heidenreich, "Obesity and Survival in Patients with Heart Failure and Preserved Systolic Function: A U-shaped Relationship,” American 
Heart Journal, Vol. 159, No. 1, 2010, pp. 75-80. doi:10.1016/j.ahj.2009.10.026

[21] A. Oreopoulos, R. Padwal, K. Kalantar-Zadeh, G. C. Fonarow, C. M. Norris and F. A. McAlister, "Body Mass Index and Mortality in Heart Failure: A Meta-Analysis," American Heart Journal, Vol. 156, No. 1, 2008, pp. 1322. doi:10.1016/j.ahj.2008.02.014

[22] T. A. Paajanen, N. K. Oksala, P. Kuukasjarvi and P. J. Kahrhunen, "Short Stature Is Associated with Coronary Heart Disease: A Systematic Review of the Literature and A Meta-Analysis,” European Heart Journal, Vol. 31, No. 14, 2010, pp. 1802-1809. doi:10.1093/eurheartj/ehq155

[23] M. L. Kortelainen and T. Sarkioja, "Coronary AtheroscleRosis Associated with Body Structure and Obesity in 599
Women Aged between 15 and 50 Years,” International Journal of Obesity, Vol. 23, No. 8, 1999, pp. 838-844. doi:10.1038/sj.ijo.0800960

[24] S. P. Wamala, M. A. Mittelman, M. Horsten, K. Schenck-Gustafsson and K. Orth-Gomer, "Short Stature and Prognosis of Coronary Heart Disease in Women," Journal of Internal Medicine, Vol. 245, No. 6, 1999, pp. 557-563. doi:10.1046/j.1365-2796.1999.00454.x

[25] E. F. Philbin and T. G. DiSalvo, "Influence of Race and Gender on Care Process, Resource Use, and HospitalBased Outcomes in Congestive Heart Failure," American Journal of Cardiology, Vol. 82, No. 1, 1998, pp. 76-81. doi:10.1016/S0002-9149(98)00233-1 